\title{
TRANSDISCIPLINARIDADE DO DESIGN: NÍVEIS DE REALIDADE DISTINTOS
}

\section{DESIGN TRANSDISCIPLINARITY: DIFFERENT LEVELS OF} REALITY

\section{Sónia Liliana da Silva Vieira ${ }^{1}$}

RESUMO: A partir das noções de disciplina, design e transdisciplinaridade, este artigo faz uma revisão da literatura sobre design transdisciplinar e desdobra perspetivas sobre e para seu desenvolvimento a partir da análise e observação decorrentes de casos de estudo. O design como disciplina engloba diversas áreas de projeto, anfitriãs do design, que nutrem o espaço particular de cada uma e se sobrepõem em espaços partilhados, nos quais as suas influências particulares são visíveis em abordagens e artefatos resultantes. Partindo do three space model, da revisão da literatura apresentada e da observação e estudos empíricos em ambientes inter, multi e transdisciplinares, este artigo evidencia características da influência do design na dinâmica dos seus espaços transdisciplinar, partilhado e particular. Para além da identificação dos aspetos particulares e partilhados das áreas de projeto, os aspetos comuns têm sido um dos desafios do design transdisciplinar que passa pela identificação das suas invariantes, quer sejam de natureza específica ou não específica do design, com influência transdisciplinar capaz de alcançar e influenciar outras disciplinas. Designers, investigadores e educadores almejam identificar tais características, conhecimento que, uma vez integrado, será de grande utilidade e aplicação no desenvolvimento dos processos sociais de investigação, educação e prática do design. Com o intuito de despertar para os diversos níveis da realidade da colaboração no design, designers, estudantes e demais interessados, este artigo descreve e estrutura esses âmbitos para melhor compreensão do design transdisciplinar.

\section{PALAVRAS-CHAVE: Design; Transdisciplinaridade; Ciência; Prática; Realidade.}

ABSTRACT: This paper presents literature review on the topic of transdisciplinary design from notions of design, discipline and transdisciplinarity, unfolds perspectives of its understanding and development, and identifies different levels of transdisciplinary design based on case studies analysis and observation. Design as a discipline entails diverse project areas, each one nurtures its particular space, as design host discipline. Overlapping between host disciplines happens in shared spaces in which particular influence of approach and resultant artifacts can be distinguished. Based on literature review, three space model, and from empirical studies and observation of inter, multi and transdisciplinary environments, a contribution is given to the task of bringing into evidence characteristics of the influence of design and the dynamics of its particular, shared and transdisciplinary spaces. Besides particular and shared aspects of host disciplines, identifying common aspects of design has been the defying challenge of design specific or non-specific invariants of transdisciplinary influence over other disciplines. Designers, researchers and educators aim to achieve the body of knowledge of these characteristics that can be of great use and application for the development of social processes of design practice, education and research. With the purpose of bringing awareness to designers, students and anyone interested, this paper structures several levels of reality and collaboration in design, for a better reflection on its transdisciplinarity.

KEYWORDS: Design; Transdisciplinarity; Science; Practice; Reality. 


\section{DISCIPLINA, TRANSDISCIPLINARIDADE E DESIGN}

Disciplina é uma atividade da experiência que proporciona determinado treino físico e mental, assim como o seu avanço numa área do conhecimento (por exemplo, design). As contribuições na literatura para definir o design como disciplina (ARCHER, 1979) focam aspectos como considerações, valores e propósitos do design como domínio de extensa influência, e a emergente necessidade de organizar e estruturar o conhecimento científico acerca do design (CROSS, 2001; LOVE, 2002). Várias têm sido as dificuldades na consolidação do entendimento do design como disciplina. Ao longo da história da investigação em design, contribuições relevantes reivindicaram uma ciência do design (CROSS, 2001; SIMON, 1969; FULLER, 1963), contudo as dificuldades em aceder ao seu núcleo central de conhecimento e alcançar sua estrutura e organização têm sido apresentadas (LOVE, 2002; VISSER, 2009); essas dificuldades se evidenciaram com a chamada para estender a prática do design a outras áreas da ciência e atividade humana pelo interesse nas suas características inerentes (BOLAND JUNIOR; COLLOPY, 2004). A transferência do conhecimento sobre design para além da sua disciplina tem desafiado investigadores, educadores e praticantes na fundação organizada desse conhecimento emergente. Por outro lado, a progressão do uso dos termos inter, multi e transdisciplinaridade no design, que emergiu nas ultimas décadas, não é consensual nas suas definições nem aplicação, o que revela a necessidade de uma forma mais holística e completa para compreender o campo de aplicação do design no seu todo. O design cada vez mais preenche os discursos de áreas sem tradição na disciplina, através do conceito de design thinking premissas do design têm chegado a áreas como as neurociências e universidades como Harvard, depois de passar pela gestão, marketing, psicologia, entre outras. Falta uma compreensão global e transdisciplinar do design para que o presságio de Bonsieppe (1992) seja realidade: o design como a nova disciplina fundamental do século XXI, substituindo a matemática no papel transdisciplinar e fundacional que teve na ciência a partir do século XVII.

O termo transdisciplinaridade foi formalmente introduzido em 1970, no workshop internacional L'interdisciplinarité - Problemes d'enseignement et de recherch dans les universités, organizado pela Organização para a Cooperação e Desenvolvimento Económico (OCDE) em Nisa, Portugal, onde o conceito foi mencionado durante as conversas entre Jean Piaget, Erich Jantsch e André Lichnerowicz (POPESCU, 2014). Mais tarde, em 1987, com a criação do International Center for Transdisciplinary Research (CIRET) e do $1^{\circ}$ Congresso Mundial sobre Transdisciplinaridade (que aconteceu em 1994, no Convento da Arrábida, Portugal), emergiram os catorze princípios fundamentais e orientadores da investigação transdisciplinar dos quais alguns são aqui referidos pela sua direta aplicação no design, entendido como âmbito geral das disciplinas de projeto, cuja aplicação transcende os limites da sua própria disciplina. O termo transdisciplinaridade tem na história da ciência registos com cerca de cinquenta anos (GERICKE et al., 2018). Transdisciplinaridade é um tema que faz ressurgir no tempo aspectos de integração de perspetivas, e reemerge no início do século XXI pela necessidade da partilha de problemas, por vezes complexos, e de ter sido constatada a falta de convergência de conhecimento para a sua resolução, o que passa pela integração de perspetivas na formulação e resolução do problema partilhado.

Na sequência de emergentes necessidades sociais e do cruzamento das ciências, práticas e saberes para a resolução de problemas cuja novidade e complexidade fazem emergir novos tópicos carentes de construção de conhecimento, o conceito de transdisciplinaridade tem sido estendido ao design em áreas como HCI (Human Computer Interaction), service design, mix media digital, smart cities and systems, entre outras, cuja essência transdisciplinar tem levado à criação de cursos de mestrado específicos (i.e. Parson School), assim como ao crescente estudo dos seus aspectos cognitivos, 
de interesse para diversas áreas como neuromarketing, healthcare systems, experience design e de CPS (Cyber Physical Sytems) (VAN DER VEGTE; VROOM, 2018).

- Ao mesmo tempo, a emergência sentida pelo design transdisciplinar revelou-se uma oportunidade para melhor compreender e integrar a prática e o corpo de conhecimento do design entre e para além das suas disciplinas anfitriãs. As perspetivas correntes acerca da transdisciplinaridade do design e suas implicações desdobram-se nas seguintes considerações:

- requer transferência de conhecimento que ainda não é consensual na comunidade científica;

- tal transferência implica a tradução de aspectos essenciais do design que sejam de valor para a área de aplicação;

- requer desenvolvimento de novas competências, funções, pesquisa e métodos experimentais.

Como muito deste conhecimento latente está por desenvolver, as opiniões dividem-se entre alguns investigadores e designers que manifestam preocupação por uma possível perda de coesão da disciplina do design e excessiva adoção de tarefas adicionais às do design. Outros facilitam a oportunidade de transferência do design, entendido como cognição que pode ser apreendida através da experiência e desenvolvimento pessoal, extensiva a todos os campos da ação, assumindo que o design constitui o ser humano (KRIPPENDORFF, 2006), e tem, portanto, aplicação e campo de estudo em qualquer área da atividade humana.

O conceito de transdisciplinaridade foi estendido ao design, ação que teve como marco fundamental o workshop internacional The future of transdisciplinary design, organizado pela Université du Luxemburg em 2013. O design transdisciplinar surge da identificação da necessária integração de perspetiva evidenciada pela necessidade de ultrapassar dificuldades como: colaboração ineficaz; entendimento das diferentes linguagens associadas a diferentes formações de base e culturas inerentes na prática do projeto (i.e. engenharia, arquitetura, design gráfico, de produto, de interação, de serviços, da experiência); transparência na comunicação e explanação de vocabulário específico e terminologia; barreiras à colaboração inter, multi e transdisciplinar, na incerteza do papel de cada um e suas contribuições; despertar a percepção para o entendimento das diversas perspectivas em equipa constituída por pessoas com formações e funções base em diversas disciplinas do design ou além deste (marketing, vendas, estratégias de negócios, biologia, neurociências, entre outras); criação de novos conhecimentos; entendimento da complexidade, da gestão e da colaboração.

\section{CIÊNCIA, ACADEMIA E PRÁTICA DO DESIGN: ENQUADRAMENTO TRANSDISCIPLINAR}

O desajuste entre os avanços na prática do design e a sua investigação tem sido reconhecido (GERICKE; BLESSING, 2012) e a metodologia de design académica revela incongruência quando aplicada ao design no presente e futuro (BIRKHOFER, 2011). A reemergente chamada para a ciência do design (CROSS, 2001; FARRELL; HOOKER, 2013; GALLE; KROES, 2014; 2015), construções de métodos para aplicação generalizada, novos paradigmas e 
novas aplicações, métodos para a compreensão das características centrais do design como ciência pura e como ciência aplicada, mais uma vez clama pela investigação e clarificação das diferenças e semelhanças no design (GOEL; PIROLLI, 1989, 1992; VISSER, 2009), ao longo das suas disciplinas anfitriãs (LOVE, 2002), nos designers, situações, e além da própria disciplina do design.

Com o intuito de compreender o design como disciplina e construir a partir de publicações anteriores, considerando o modelo dos três espaços da influência do design - Three Space Model - (VIEIRA, 2018), esta contribuição propõe ao leitor imaginar a disciplina do design desdobrada em três esferas de atividade e conhecimento, nomeadamente, considerando-o como disciplina científica, académica e prática.

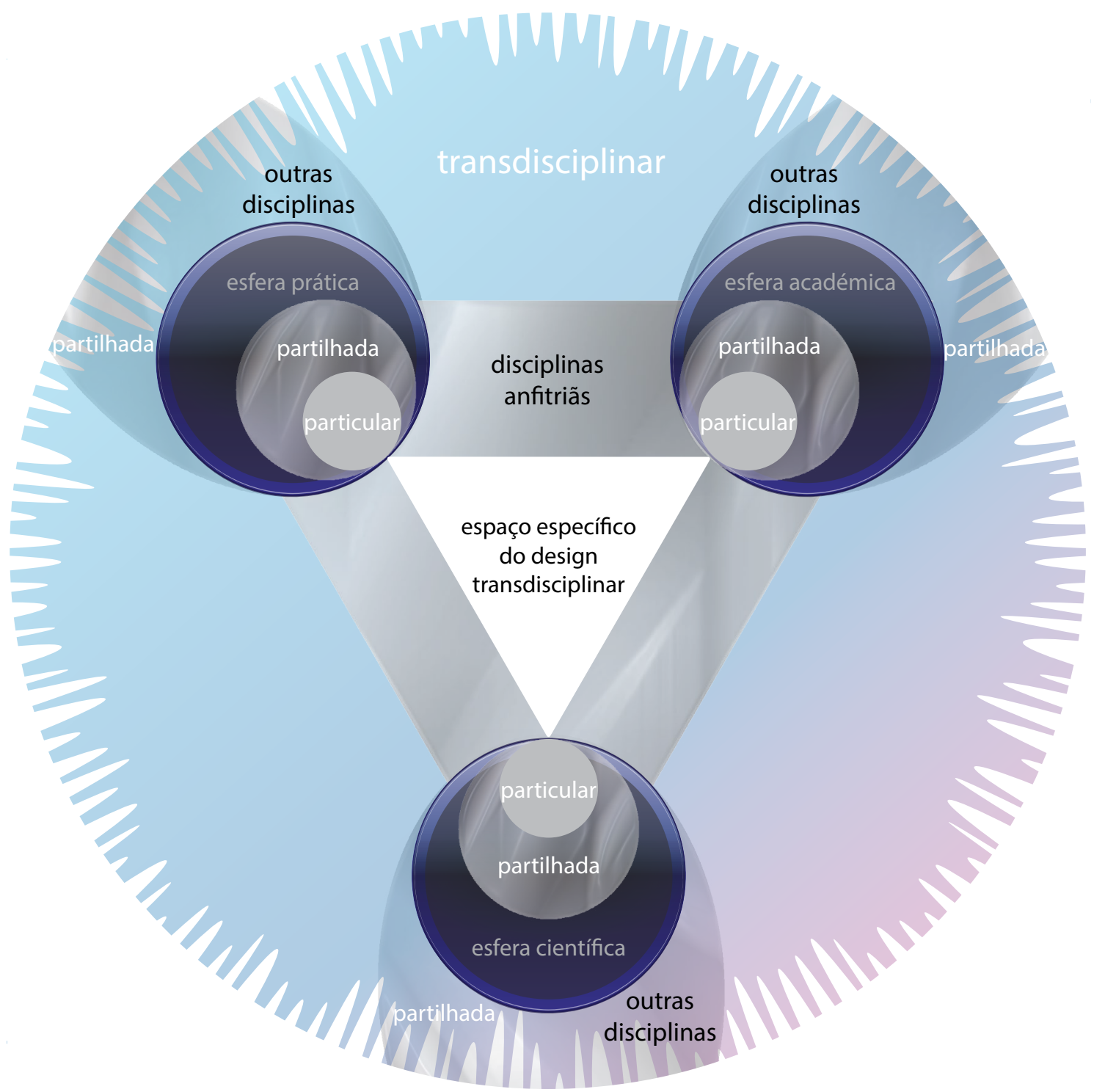

Figura 1: Representação atual do modelo dos três espaços da influência do design.

Fonte: Sónia Vieira (2018).
O design como disciplina científica é uma área do conhecimento que procura estruturar e sistematizar a compreensão sobre o design através de métodos generalizáveis e apropriados de investigação. $\mathrm{O}$ design como disciplina académica, e para propósitos educacionais, é desenvolvido em várias áreas do conhecimento, como cultura e experiência do design, projeto 
e metodologia. Enquanto o design como disciplina prática é enraizado em várias práticas profissionais e em suas disciplinas anfitriãs, clássicas, estabilizadas, novas ou emergentes, como o design gráfico, design de interação, arquitetura, engenharia, software, design de controle, design de serviços, entre outras. Este modelo permite o enquadramento e a visibilidade do espaço transdisciplinar do design, assumindo que é constituído por características do design, específicas ou não específicas, que são largamente partilhadas e, portanto, invariantes a todas as disciplinas anfitriãs, designers, situações e extensíveis a outras disciplinas e áreas partilhadas, como os ambientes de design que integram atividades multidisciplinares (design, biologia, literatura, marketing, gestão) e interdisciplinares (design industrial, arquitetura, engenharia); uma oportunidade de trazer novas perspetivas e desenvolvimentos para aceder, organizar e estruturar os resultados obtidos a partir da investigação da ciência do design. O design transdisciplinar vem reforçar a chamada para a intervenção no espaço transdisciplinar, por exemplo através da action research (ARGYRIS, 1994) em áreas não familiares. Assim sendo, os ambientes de latente transdisciplinaridade são os apropriados para explorar e construir plataformas de intervenção e entendimento dentro e além do design como disciplina, na sua mais ampla aplicação prática, acepção teórica e potencial construção científica.

\section{AMBIENTES DE DESIGN INTER, MULTI E TRANSDISCIPLINARES}

Os ambientes da prática quotidiana do design têm sido reconhecidos como apropriados para o estudo do design (MCDONNELL et al., 2009). O acompanhamento do que se passa na prática do design é central para o seu desenvolvimento como disciplina académica e científica. Métodos partilhados de investigação têm sido propostos para a construção de teoria (GERO, 2010). Da recente adoção de técnicas e métodos das áreas da psicologia cognitiva e neurociências aplicadas, por exemplo, ao neuromarketing, desenvolvimento de jogos e interfaces, mas sobretudo em estudos cognitivos de designers, esperamse plataformas de partilha de dados consolidados e validados para acesso, troca, validação e replicação desses estudos, o que tem sido fator de atraso para consolidar o design como ciência. Com a criação de plataformas de dados, sobreposição de análises e afinação dos métodos científicos, a investigação em design terá a oportunidade de deixar de ser vista como anedótica aos olhos de muitos cientistas. Com um esforço concentrado na partilha e construção conjunta de conhecimento, a investigação em design poderá deixar de ser apenas um conjunto de contribuições individuais e dispersas, sem conexões consolidantes entre si. Para a validação de uma ciência do design é necessário olhar para outras ciências e sua evolução, perceber em que estágio o design se encontra e como gerar mudanças de paradigmas e mentalidades, sem perder 0 caráter essencial da diversidade na transdisciplinaridade do design.

Resultados de estudos anteriores evidenciam alguns aspetos relevantes do design aqui referidos. Períodos de observação em quatro empresas propiciaram os contextos sociais e a oportunidade para estudar e identificar tais características (VIEIRA, 2013). Estas empresas são baseadas no design gráfico e de comunicação, no design de interatividade, na arquitetura e urbanismo, e na engenharia mecânica e mecatrónica. Partilham ambientes interdisciplinares com interações multidisciplinares externas, os casos de estudo em arquitetura e design gráfico, enquanto os casos de estudo em design de interação e engenharia usufruem de ambientes transdisciplinares. Nestes ambientes, a influência mútua e processos de troca entre os departamentos de vendas, marketing, investigação, design e desenvolvimento de produto proporcionou o ambiente apropriado para compreender a necessidade de estender as fronteiras da investigação no design para uma abordagem de estudo transdisciplinar.

Os dados foram recolhidos através de observação de projetos em curso e entrevistas a pessoas com diferentes atividades e formação-base, como 
design gráfico, interação, editorial, comunicação social, literatura, ilustração, biologia, engenharia civil, ambiental, eletrónica e aeroespacial, a gestão de projeto e de vendas. A estrutura organizacional responsável pelo design é de cerca de dez pessoas por caso de estudo, lideradas por experts, sendo que todos os casos correspondem a empresas com reputação no mercado, de onde o estudo do comportamento de designers deriva de ação competente. São também ambientes onde os designers se sentem envolvidos e têm liberdade de expressão, o que permitiu analisar os seus testemunhos com confiança. Os estudos focaram-se na identificação de variantes e invariantes do design para fins e objetivos de investigação específicos (VIEIRA, 2013). Como as invariantes do design são difíceis de aceder, seu reconhecimento foi focado na análise de descrições e comportamento humano bem-sucedido. A compreensão dos diferentes casos de estudo, suas culturas, filosofias de design, métodos específicos, ferramentas e sequência de práticas levou à conclusão de que o design ao longo das suas disciplinas e âmbitos de implementação tem diferentes níveis de influência (VIEIRA, 2018).

\section{BASES PARA A TRANSDISCIPLINARIDADE DO DESIGN}

Dos catorze princípios enumerados no $1^{\circ}$ Congresso do CIRET (1994), oito são aqui destacados: a transdisciplinaridade como conceito que se expande para além do campo das ciências exatas, e para a reconciliação com as humanidades e ciências sociais, arte, literatura, poesia e experiência espiritual; a transdisciplinaridade multireferencial e multidimensional, tendo em conta as várias abordagens no tempo e na história; a transdisciplinaridade que não sobrevaloriza o conhecimento abstrato, mas considera abordagens contextuais concretas e globais; a educação transdisciplinar que revaloriza a intuição, a imaginação, a sensibilidade e o corpo na transmissão de conhecimento; o diálogo e a discussão no respeito pelo indivíduo e pelo coletivo, na partilha do conhecimento e na sua apreensão, com rigor abertura e tolerância; a economia transdisciplinar ao serviço do ser humano e não o reverso; o respeito pelos diferentes tipos de lógica associados ao pensamento; e por fim a unificação semântica e prática dos significados que atravessam e vão além das disciplinas (NICOLESCU, 2002).

A transdisciplinaridade é entendida como complementar às abordagens disciplinares e emerge de novos dados, fatos e interações. Nicolescu (2008) define transdisciplinaridade a partir de três postulados, sendo que na presença de distintos níveis de realidade, o espaço não só entre disciplinas, mas que as transcende está repleto de informação. Transdisciplinaridade é sobre a transgressão de fronteiras entre disciplinas (NOWOTNY, 2006) para a integração de diversas formas de conhecimento, prática, saber e investigação.

Uma hipótese designada design genérico, proposta por Goel e Pirolli(1989), clama pelo estudo do design como matéria por si só. Esta proposta holística identifica o design como problem-solving distinto ao longo de disciplinas específicas. Vinte anos depois, Visser (2009) estende a hipótese inicial e identifica o design como um distinto processo cognitivo. Nesta contribuição, a autora estrutura linhas de necessária investigação e evidência empírica, considerando as seguintes premissas: diferentes disciplinas anfitriãs do design partilham grandes aspetos comuns; as disciplinas anfitriãs do design mostram variação nas semelhanças; as abordagens dos designers variam ao longo e dentro das disciplinas anfitriãs do design; as situações criam variação na cognição dos designers; o design é diferente do não design.

Várias contribuições na literatura demonstram semelhanças e diferenças entre disciplinas do design ou não. Aspectos relevantes têm sido abordados, tais como: semelhanças e diferenças nos processos cognitivos em diferentes áreas do design (AKIN, 2001; GERO, 2010), diferenças na terminologia (REYMEN, 2001), formas particulares do conhecimento dos designers (CROSS, 2006), os artefatos que caracterizam as disciplinas como sendo variantes nas 
diferentes disciplinas do design (HUBKA; EDER, 1987), entre muitas outras, contudo, contribuições dispersas. Sendo a identificação de variantes e invariantes um dos propósitos da ciência (SIMON, 1990), outras contribuições apresentam resultados acerca do design que passamos a descrever.

\section{Invariantes}

O design é considerado um tipo de atividade cognitiva, e não apenas status profissional (SIMON, 1969; JONES, 1979; VISSER, 2009). O espaço do problema e o espaço da solução progridem em coevolução (DORST; CROSS, 2001). O design envolve problemas complexos que são raramente decompostos em subproblemas independentes (SIMON, 1973; AKIN, 2001; GOEL, 1995), e é também uma atividade 'satisficing' em que não se procura a solução otimizada (SIMON, 1987). Os designers geram uma solução estruturante inicial (DARKE, 1979; CROSS, 2001). Os problemas do design têm várias soluções aceitáveis (EASTMAN, 1970) que, com os critérios de avaliação e os procedimentos, conduzem a avaliação (LERA, 1981; D’ASTOUS et al., 2004). Os designers reutilizam o conhecimento através de raciocínio analógico (CASAKIN; GOLDSCHMIDT, 1999), e a atividade do design é maioritariamente organizada pelo reconhecimento de oportunidades (VISSER, 1994; 2009).

O design é uma atividade de resolução de problemas (NEWELL, 1972; SIMON, 1973), que são considerados ill-defined (SIMON, 1973; THOMAS; CARROLL, 1979). Tendo em conta que algumas destas questões relevantes, polémicas e por vezes divergentes são ainda passíveis de validação contrária, devem portanto ser consideradas não consensuais (FARRELL; HOOKER, 2013) e não específicas, uma vez que estão também presentes em outras atividades. Da revisão da literatura e de sugestões plausíveis que surgiram durante estudos empíricos surgiu a evidência de que, por exemplo, algumas destas características invariantes não são específicas do design, e o que é específico do design é também invariante ao longo e além das suas disciplinas.

Dos estudos realizados, as seguintes invariantes foram constatadas: os casos de estudos são baseados em disciplinas cujos processos de design passam pela materialização de ideias com efeitos tangíveis e intangíveis; as características dos processos de iteração no design são comuns, assim como as características dos processos de interdependência de suas questões; a influência da iteração e interdependência de assuntos na tomada de decisão é universal (VIEIRA et al, 2011a; 2013); o modelo de fruição da interação em reuniões de design entre stakeholders é comum (VIEIRA, 2011b, 2013), sem apresentar elementos específicos.

Existem invariantes das abordagens dos designers (VIEIRA et al., 2010a) que fazem convergir consubstancial consideração e esforço na formulação de ideias e soluções, para as quais são especificados atributos, propriedades e qualidades. Os problemas complexos, por sua vez, fazem convergir designers de diferentes formações e com diferentes abordagens na resolução dos problemas, partilhando a abordagem criativa e o contexto. Para cada formação de base, características particulares têm papel crucial e influenciam a abordagem e as ações dos designers. Contudo colaborar e partilhar o processo de design requer o entendimento de tarefas de gestão e partilha, e comunicação de ricos conjuntos semânticos. A materialização da semântica toma diferentes formas e representações ao longo das diferentes disciplinas anfitriãs do design existentes em cada equipa.

Quer sejam específicas ou não, as invariantes do design têm influência transdisciplinar, especialmente as que constituem comportamento na troca em processos de negociação, de interdependência e tomada de decisão. As invariantes específicas do design relacionam-se com processos de avaliação, flexibilidade processual, questões de design e características da abordagem. O que é transdisciplinar é não só invariante ao longo e para lá da disciplina do design, mas pede identificação clara, transferência e tradução, focada nos aspectos essenciais para cada área de aplicação. 


\section{Invariantes específicas}

Tem sido de grande debate e grau de dificuldade a identificação das características específicas do design. Contudo, algumas premissas têm sido apontadas, nomeadamente, estruturas e atividades cognitivas específicas do design (VISSER, 2009), formas próprias de conhecimento dos designers (CROSS, 2002), a expertise no design considerada específica (LAWSON; DORST, 2009). Um aspecto chave é o chamado 'problem framing' (CROSS, 2004; DORST, 2015), expert designers são focados na solução mais do que no problema (CROSS, 2006), de que existe um modo de pensar e comunicar design (JONES, 1979; LAWSON; DORST, 2009). Para além da mais recente perspectiva da hipótese sobre design genérico (VISSER, 2009), que clama por uma vasta e alargada evidência empírica, emerge a validação e compreensão das relações entre estas características para a construção do corpo do conhecimento do design. Outras contribuições da literatura apresentam também linhas orientadoras e clamam pela evidência empírica das variantes, invariantes e especificidades do design (GOEL; PIROLLI, 1992; AKIN, 2001; SIMON, 1990). Acaloradas discussões em periódicos (FARRELL; HOOKER, 2013; GALLE; KROES, 2014; 2015) despertaram para o sentido crítico acerca de prévias assunções não absolutas e, portanto, contestáveis, que têm sido adotadas com prejuízo para o desenvolvimento da investigação em design. Tal compreensão requer clareza generalizada e consensual acerca das fronteiras e limites do design, suas disciplinas anfitriãs, terminologia, conceitos base, cultura, contextos e situações específicas. Mais recentemente, vários estudos que integram métodos da psicologia cognitiva e em particular das neurociências, como FMRI (Functional Magnetic Ressonance Imaging) e EEG (Electroencefalograma), começam a surgir em conferências e periódicos na tentativa de alcançar uma validação robusta e fundamentada acerca das especificidades do design (ALEXIOU et al., 2009), detectadas ao nível das ondas cerebrais, áreas ativadas no cérebro e sua dinâmica no desempenho de atividades de design e outras.

Nos estudos empíricos realizados, as seguintes invariantes específicas foram constatadas, embora, como é de esperar, a dificuldade de aceder e validar a especificidade de caraterísticas relativas ao design seja de alto grau. Contudo, desenhar ação em situações concretas e delas reconhecer linhas orientadores para a construção de significado através do design, é uma invariante transdisciplinar, isto é, extensível a outras práticas da atividade humana. Atenção e cuidado são dados à situação concreta em contexto social, e os designers adotam atitudes interdependentes e assumem eixos multidirecionais de comportamento para a identificação e reconhecimento da ação situada para ser específica a implementação e concretização da solução (VIEIRA et al., 2010b). A análise de valor e tomada de decisão são invariantes do comportamento dos designers que desempenham um papel interdependente e crucial no design como processo social e individual (VIEIRA et al., 2010a), em particular, os designers dão prioridade a valores nas tomadas de decisão que têm por base emoções, intuições, razões, experiência e constrangimentos; cinco categorias invariantes nos designers. Os elementos dos mecanismos para gerir situações de crise (VIEIRA, 2013) são também invariantes, e as suas estruturas relacionais apresentem maior flexibilidade do que os não designers.

\section{Invariantes de representação variável}

As variantes do design têm sido identificadas no processo e as estratégias adotadas são: ferramentas em uso, tarefas de rotina, o papel do utilizador no processo do design, a diversidade entre os designers e a variação na expertise (KRUGER; CROSS, 2006; BALL; CHRISTENSEN, 2009; CHRISTENSEN; BALL, 2016; VISSER, 2009; LAWSON; DORST, 2009), idiossincrasia inerente, por exemplo, à cultura de design adotada, variação na inserção política, económica, antropológica e social (VISSER, 2009), variação na evolução dos 
artefatos e tipos de artefatos (HUBKA; EDER, 1987). Para cada disciplina anfitriã do design, características particulares têm papel crucial e influenciam as ações e abordagens dos designers (VIEIRA et al., 2010b). Tal fato é suportado pelo conceito de object-worlds (BUCCIARELLI, 2003), a ideia de que diferentes participantes vêm o objeto de design diferentemente, dependendo da educação, formação base, treino, desenvolvimento de competências, responsabilidades e interesses metodológicos.

Nos estudos realizados, as seguintes variantes foram constatadas: condições culturais, condições regulamentares, reconhecimento de conhecimentos não desenvolvidos, identificação das emoções e sentimentos que se pretendem transmitir às pessoas, assim como seus níveis de satisfação, são características partilhadas que assumem representação variável (VIEIRA et al., 2010a; 2013). Entre elas, encontram-se outros aspectos como propósito e direção a prosseguir, escolhas de design como estilo, linguagem e abordagem pré-definidas, experiência, simulação das situações, e uma eficaz comunicação das sensações a transmitir às pessoas.

$\mathrm{O}$ natural entendimento por parte dos designers acerca das vantagens e desvantagens de usar métodos estruturados, assim como procedimentos de design adequados a cada situação e problemática revelam a flexibilidade dos designers nas abordagens metodológicas (VIEIRA, 2013).

Os profissionais da área consideram as narrativas a transmitir, o tempo e a escala, as ações e reações dos utilizadores, os movimentos e as dimensões relativas, as primeiras impressões e imagens que surgem no consciente acerca das primeiras características das soluções, a comunicação eficaz de efeitos tangíveis e intangíveis, a cor, a estética, a luz, o som, os materiais, entre outras para as escolhas que são feitas com base na prioridade, viabilidade, exequibilidade e importância. Porque os designers precisam observar seu próprio pensamento de forma objetiva.

Resultados sobre características variantes e particulares das disciplines anfitriãs com influência na abordagem de design foram também identificados (VIEIRA, 2010b; 2013). O design gráfico, por exemplo, é assunto comum a todas as outras disciplinas, tem processos rápidos, conteúdos muito variantes por briefing, é de fácil reprodutibilidade, distribuição e difusão na sociedade. É uma disciplina clássica baseada na semiótica e comunicação, estável no conhecimento e processos, mas aberta às novas tecnologias e abordagens, é comum, mas também bastante independente.

O design de interação, disciplina emergente de processos de design complexos, que envolve designers com diversas formações com assimilação de tarefas de gestão, explora e adota resultados de artesanato tecnológico, processos embrionários de reprodutibilidade, identifica conhecimentos por desenvolver para os quais há necessidade, sendo uma área de descoberta de novos assuntos de design.

A arquitetura, disciplina clássica que gere a integração de várias disciplinas, tem longos processos de design, com resultados visíveis a longo prazo, e reprodutibilidade abrandada. Por ter a função de representação social e foco particular no design de autor e permanência dos resultados, tem maior responsabilidade social e conhecimento estável.

A engenharia mecânica, também uma disciplina clássica, é fundamental para a sociedade e crítica para muitas atividades do design, quer por sua natureza regulamentar, quer pela infindável busca pela descoberta, associando ciência e tecnologia, à evolução e complexidade dos processos, quer pela responsabilidade social. Papel este que é estendido pelo design industrial, ao fazer convergir arte, ciência e tecnologia, na reprodução em massa de conteúdos variáveis, com foco nas questões da usabilidade, utilidade social e desejo de propiciar melhores e agradáveis experiências. Sempre com processos de design longos e interconectados com muitas outras áreas do conhecimento.

Variantes do design podem ter uma influência parcialmente partilhada ou particular nos designers, ambientes de design e disciplinas anfitriãs. Algumas variantes têm influência particular e caracterizam as disciplinas 
anfitriãs ou abordagens de design. Outras variantes, parcialmente partilhadas entre designers, relacionam-se com a atividade profissional e afinidade. Por exemplo, os métodos da engenharia do design são analíticos, baseados no cálculo, variáveis e otimização. Enquanto que os métodos do design industrial são baseados na síntese, clarificação, investigação estruturada, discussão e análise de decisões. A abordagem dos engenheiros é hierárquica e matemática, baseada na ciência e teoria, conceitos e princípios, causa e efeito, medição, estimativa e certeza. O design é um processo social de negociação, interação, de retificação em que por vezes até mal-entendidos contribuem para um processo rico em ambiguidade e incerteza. Cada disciplina tem o seu conjunto de características particulares, alguns constituem inteiramente partes de conjuntos maiores, alguns conjuntos sobrepõem-se, outros são disjuntos, isto é, não contêm elementos em comum. As áreas de sobreposição entre disciplinas são, no seu conjunto de características, também uma unidade reconhecida.

\section{DISCUSSÃO}

O entendimento da transdisciplinaridade do design representa uma estrutura mais complexa, e ao mesmo tempo mais subtil, mas também necessária para que a mente humana interessada consiga abarcar o campo de ação do design. Do reconhecimento desta estrutura complexa, e necessidade de uma melhor clarificação, fica para já o sentimento de que é importante iluminar as relações entre as partes, para as quais será necessário assumir o estudo da dinâmica da estrutura transdisciplinar do design. Talvez até alcançar padrões não só de influência, mas também de controle, rutura, mudança de paradigma, fecho e abertura da área partilhada ou disciplina, assim como identificação de tensões sentidas, que levam à mudança das relações entre as partes, assim como à renovação de velhos padrões de sobreposição que passam a ser inapropriados e dão espaço a novos padrões, por vezes caóticos outras vezes estruturados, acompanhando assim a evolução das necessidades sociais. A sobreposição que ocorre nos espaços partilhados é um regenerador vital da estrutura. Cada disciplina ou espaço partilhado encontra o seu lugar e cria a sua atmosfera e influência, sobrepondo-se os aspectos que foram absorvidos e que se tornaram predominantes. Os espaços parcialmente partilhados entre disciplinas do design são híbridos de maior liberdade criativa, muito focados na comunicação (teatro, cinema, animação, documentário, media digital). Realidades distintas caracterizam os espaços particulares de cada disciplina do design, escolas, empresas e culturas, ao mesmo tempo o espaço transdisciplinar que atravessa todas as disciplinas do design, e que lhes permite a extensão e aplicação em outras áreas da atividade humana e integração por outras disciplinas científicas, académicas e da prática, é de natureza expansiva. É no espaço transdisciplinar onde melhor se identificam as oportunidades de novas aplicações do design, tendências emergentes, as invariantes e onde os produtos do design atingem as massas. No espaço transdisciplinar, fatores como a extensão e consequente absorção de novas tecnologias, leis e regulamentos que pedem novas abordagens em disciplinas clássicas do design, assim como a tradução de características específicas do design em outros estados para troca, expansão e inclusão do design transdisciplinar, são aspetos cruciais a desenvolver. Mudança de métodos, padrões de pensamento e a habilidade para comunicar ao longo das profissões do design são identificados assim como também representam competências a desenvolver para o entendimento e receptividade a outras e diferentes perspetivas dos processos de design.

Da análise do espaço transdisciplinar, parcialmente partilhado e particular da influência das invariantes, variantes, e características específicas do design, revela-se uma reestruturação da chamada para evidência empírica na investigação em design. Nesta contribuição, 
juntaram-se propostas investigadoras e académicas, de agentes e designers, e foram identificados aspectos essenciais do design, portanto, características específicas e invariantes referidas na literatura. Do que podemos constatar, o design para lá da sua disciplina tem um nível de influência baseado em invariantes partilhadas e específicas. Algumas dessas características são largamente partilhadas entre designers e não designers (i.e. processos de decisão e de iteração). As invariantes específicas, algumas referidas na literatura, de difícil acesso e identificação, são alcançáveis num nível abstrato e qualitativo. As disciplinas anfitriãs do design sobrepõem-se umas às outras em espaços comuns e partilhados, e influência particular é visível em abordagens dorsais que resultam em artefactos distintos. No espaço parcialmente partilhado são identificadas características divididas entre grupos de designers, tais como designers com base na engenharia (mecânica, software, industrial), designers com base nas artes (gráfico, arquitetos), designers com base no design (interação, experiência, serviço, produto), que estruturam os âmbitos e influenciam as abordagens de design e resultados em ambientes interdisciplinares e multidisciplinares. Estes espaços híbridos permitem maior liberdade criativa, incluindo a criação de novas disciplinas do design. No espaço particular, é possível identificar as características das disciplinas anfitriãs, como cultura, problemas de design, situações e características das abordagens ao design, assim como o design de intangíveis (JONES, 1979) que envolve características particulares por disciplina de design, a criação de disciplinas emergentes tais como design de serviço com influência interdisciplinar. Porém, mais pode e deve ser feito.

Em essência, o design envolve ações mentais e físicas que podem ser transdisciplinares, parcialmente partilhadas ou particulares aos designers ao longo e para lá da sua disciplina. Designers, investigadores e educadores têm como incumbência identificar tais características para o ensino e desempenho da gestão destas ações no processo social da investigação no design, educação e prática.

\section{CONCLUSÕES}

Compreender os processos subjacentes ao design em contextos transdisciplinares pode ajudar a identificar invariantes do design e características específicas. Tal identificação pode melhor suportar a extensão da prática do design a outras áreas da atividade humana e assim ultrapassar a presente dificuldade da transferência do conhecimento em design para lá da sua disciplina. Dos estudos no design como um todo e das suas unidades, a comparação ocorre numa mais vasta e interconectada rede social e política, de contextos económicos de forças mútuas e influentes. Tais contextos requerem uma plataforma para uma mais consciente compreensão apoiada por desenvolvimentos líderes, possibilitando uma orientação que é flexível e aplicável ao longo de áreas onde o design pode ter influência. A criação e desenvolvimento de soluções metodológicas, técnicas e novas tecnologias para a melhoria das condições humanas, focadas em questões cruciais e latentes como a redução dos impactos ambientais, económicos e sociais, é premente, e só pode acontecer em ambientes e equipas não apenas multi, mas transdisciplinares. Daí a importância de nutrir uma cultura de receptividade, abertura e inovação, para ultrapassar e resolver os problemas complexos que o presente e o futuro nos colocam.

Neste sentido, esta contribuição deixa como mote final a proposta da criação de uma plataforma de investigação no espaço transdisciplinar, constituída por grupos de investigação interconectados baseados em diferentes disciplinas do design. A investigação transdisciplinar por disciplina pode antecipar e abrir caminho para a identificação de problemas inter, multi e transdisciplinares, dificuldades, barreiras, lacunas na investigação, funções e métodos por desenvolver. A implementação de tal plataforma poderia beneficiar a adoção de métodos e abordagens da 
investigação em ação, com influência em contextos de intervenção, e assim beneficiar a criação de causas para o desenvolvimento do conhecimento.

\section{AGRADECIMENTOS}

Este estudo foi desenvolvido no âmbito de pós-doutoramento financiado pela Fundação para a Ciência e a Tecnologia, com a referência SFRH/ BPD/104281/2014, na instituição INEGI-FEUP.

\section{REFERÊNCIAS}

AKIN, Ö. Variants in design cognition. In: EASTMAN, C.; MCCRAKEN, M.; NEWSTETTER, W. (Eds.). Design knowing and learning: cognition in design education. Amsterdam: Elsevier, 2001. p. 105-124.

ALEXIOU, K. et al. Exploring the neurological basis of design cognition using brain imaging: some preliminary results. Design Studies, Amsterdam, v. 30, n. 6, p. 623-647, 2009.

ARCHER, B. Design as a discipline. Design Studies, Amsterdam, v. 1, n. 1, p. 17-20, 1979.

ARGYRIS, C. Knowledge for action: a guide to overcoming barriers to organizational change. San Francisco: Jossey-Bass, 1994.

BALL, L. J.; CHRISTENSEN, B. T. Analogical reasoning and mental simulation in design: two strategies linked to uncertainty resolution. Design Studies, Amsterdam, v. 30, n. 2, p. 169-186, 2009.

BIRKHOFER, H. Introduction. In: BIRKHOFER, $H$. (Ed.). The future of design methodology. London: Springer-Verlag, 2011. p. 1-18

BOLAND JUNIOR, R.; COLLOPY, F. Managing as designing. Palo Alto: Stanford University Press, 2004.

BONSIEPPE, G. Teoria e prática do design industrial: elementos de um manual crítico. Lisboa: Centro Português do Design, 1992.

BUCCIARELLI, L. Engineering philosophy. Delft: Delft University Press, 2003.

CASAKIN, H.; GOLDSCHMIDT, G. Expertise and the use of visual analogy: implications for design education. Design Studies, Amsterdam, v. 20, n. 2, p. 153-175, 1999.

CHRISTENSEN, B. T.; BALL. L. J. Dimensions of creative evaluation: distinct design and reasoning strategies for aesthetic, functional and originality judgments. Design Studies, Amsterdam, v. 45, part A, p. 116-136, 2016.

CROSS, N. Designerly ways of knowing: design discipline versus design science. Design Issues, Cambridge, MA, v. 17, n. 3, p. 49-55, 2001.
Creative cognition in design: processes of exceptional designers. In: HEWETT, T. et al. (Eds.). Creativity and cognition: proceedings of the fourth conference on creativity and cognition. New York: ACM, 2002. p. 14-19.

Expertise in design: an overview. Design Studies, Amsterdam, v. 25, n. 5, p. 427-441, 2004

\section{Designerly ways of knowing.}

London: Springer-Verlag, 2006.

DARKE, J. The primary generator and the design process. Design Studies, Amsterdam, v. 1, n. 1, p. 36-44, 1979.

D'ASTOUS, P. et al. Changing our view on design evaluation meetings methodology: a study of software technical review meetings. Design Studies, Amsterdam, v. 25, n. 6, p. 625-655, 2004.

DORST, C.; CROSS, N. Creativity in the design process: co-evolution of problemsolution. Design Studies, Amsterdam, v. 22, n. 5, p. 425-437, 2001.

EASTMAN, C. M. On the analysis of intuitive design processes: emerging methods in environmental design and planning. Cambridge, MA: MIT Press, 1970.

FARRELL, R; HOOKER, C. Design, science and wicked problems. Design Studies Amsterdam, v. 34, n. 6, p. 681-705, 2013.

FULLER, R. B. World design science decade, 1965-1975: five two-year phases of a world retooling design proposed to the international union of architects for adoption by world architectural schools. Carbondale: Southern Illinois University, 1963.

GALLE, P; KROES, P. Science and design: Identical twins? Design Studies, Amsterdam, v.35, n.3, p. 201-231, 2014.

Science and design revisited. Design Studies, Amsterdam, v. 37, p. 67-72, 2015.

GERICKE, K.; BLESSING, L. An analysis of design process models across disciplines. In: 
INTERNATIONAL DESIGN CONFERENCE, 12., Dubrovnik. Proceedings... Zagreb: Faculty of Mechanical Engineering and Naval Architecture; University of Zagreb, 2012. p. 171-180.

GERICKE, $K$. et al. Opening up design methodology. In: BLESSING, L.; QURESHI, A. J.; GERICKE, K. (Eds.). The future of transdisciplinary design: proceedings of the workshop on "the future of transdisciplinary design”. London: SpringerVerlag, 2018. No prelo.

GERO, J. Generalizing design cognition research. In: DESIGN THINKING RESEARCH SYMPOSIUM, 8., Sydney. Proceedings... Sydney: DAB Documents, 2010. p. 187-198.

GOEL, V. Sketches of thought. Cambridge, MA: MIT Press, 1995.

GOEL, V.; PIROLLI, P. Motivating the notion of generic design within information-processing theory: the design problem space. Al Magazine, Palo Alto, v. 10, n. 1, p. 18-36, 1989.

The structure of design problem spaces. Cognitive Science, Medford, v. 16, $n$. 3, p. 395-429, 1992

HUBKA, V.; EDER, W. E. A scientific approach to engineering design. Design Studies Amsterdam, v. 8, n. 3, p. 123-137, 1987.

JONES, J. C. Designing designing. Design Studies, Amsterdam, v. 1, n. 1, p. 31-35, 1979.

KRIPPENDORFF, K. The semantic turn, a new foundation for design. Abingdon: Taylor \& Francis; CRC Press, 2006. p. 74.

KRUGER, C.; CROSS, N. Solution driven versus problem driven design: strategies and outcomes. Design Studies, Amsterdam, v. 27, n. 5, p. 527-548, 2006.

LAWSON, B.; DORST, K. Design Expertise. New York: Architectural Press, 2009.

LERA, S. G. Architectural designers' values and the evaluation of their designs. Design Studies, Amsterdam, v. 2, n. 3, p. 131-137, 1981.

LOVE, T. Constructing a coherent crossdisciplinary body of theory about designing and designs: some philosophical issues. Design Studies, Amsterdam, v. 23, n. 3, p. 345-361, 2002.

MCDONNELL, J. et al. About: designinganalysing design meetings. London: Taylor \& Francis, 2009

NEWELL, A. Human problem solving Englewood Cliffs: Prentice Hall, 1972.

NICOLESCU, B. Manifesto of transdisciplinarity. Albany: Suny Press, 2002.
(Ed.). Transdisciplinarity: theory and practice. New York: Hampton Press, 2008.

NOWOTNY, $H$. The potential of transdisciplinarity. Wien, 2006. Disponível em: <https://goo.gl/uw3kQL>. Acesso em: 31 ago. 2017.

POPESCU, G. From psychological-disciplinary knowledge to a transdisciplinary one. Procedia: Social and Behavioral Sciences Amsterdam, v. 128, p. 438-441, 22 Apr. 2014.

REYMEN, I. M. M. J. Improving design processes through structured reflection: a domain-independent approach. Eindoven: Technische Universiteit Eindhoven, 2001.

SIMON, H. A. The sciences of the artificial Cambridge, MA: MIT Press, 1969.

. The structure of ill-structured
problems. Amsterdam, v. 4, n. 3-4, p. 181-201, 1973.

Problem forming, problem finding, and problem solving in design. In: COLLEN, A.; GASPARSKI, W. W. (Eds.). Design and systems: general applications of methodology. Piscataway: Transaction Publishers, 1987. p. 245-257.

Invariants of human behavior Annual Reviews Psychology, Palo Alto, v. 41, p. 1-20, Feb. 1990.

THOMAS J. C.; CARROLL, J. M. The psychological study of design. Design Studies, Amsterdam, v. 1, n. 1, p. 5-11, 1979.

VAN DER VEGTE, W.; VROOM, R. Considering cognitive aspects in designing cyber-physical systems: an emerging need for transdisciplinarity. In: BLESSING, L.; QURESHI, A. J.; GERICKE, K. (Eds.). The future of transdisciplinary design: proceedings of the workshop on "the future of transdisciplinary design”. London: Springer-Verlag, 2018. No prelo.

VIEIRA, S. Crucial actions in design, a framework of awareness to critical situations: from a Lean Thinking perspective. 2013. Tese (Doutorado em Design) - Technical University of Delft, Delft, 2013.

Transdisciplinary design: the environment for bridging research across and beyond design as a discipline. In: BLESSING, L.; QURESHI, A. J.; GERICKE, K. (Eds.). The future of transdisciplinary design: proceedings of the workshop on "the future of transdisciplinary design". London: Springer-Verlag, 2018. No prelo.

VIEIRA, S. et al. Understanding how designers' thinking and acting enhance the value of the design process. In: DESIGN THINKING RESEARCH SYMPOSIUM, 8., 
Sydney. Proceedings... Sydney: DAB Documents, 2010a. p.107-120.

Substance variation in design approach. In: INTERNATIONAL DESIGN CONFERENCE, 11., Dubrovnik. Proceedings... Zagreb: Faculty of Mechanical Engineering and Naval Architecture; University of Zagreb, 2010b. p. 523-534.

Patterns of designers' thinking and acting in design meetings. In: INTERNATIONAL ASSOCIATION OF SOCIETIES OF DESIGN RESEARCH CONFERENCE, AIASDR11, 2011, Delft. Proceedings... Delft: Technical University of Delft, 2011a.
Designers' thinking and acting in meetings with clients. In: INTERNATIONAL CONFERENCE ON ENGINEERING DESIGN, ICED11, 2011, Copenhagen. Proceedings... Copenhagen: Technical University of Denmark, 2011b.

VISSER, W. Organisation of design activities: opportunistic, with hierarchical episodes. Interacting with Computers, Amsterdam, v. 6, n. 3, p. 239-274, 1994

Design: one, but in different forms.

Design Studies, Amsterdam, v. 30, n. 3, p. 187-223, 2009. 